\title{
A HYPERCYCLIC OPERATOR WHOSE ADJOINT IS ALSO HYPERCYCLIC
}

\author{
HECTOR SALAS
}

(Communicated by Palle E. T. Jorgensen)

\begin{abstract}
An operator $T$ acting on a Hilbert space $H$ is hypercyclic if, for some vector $x$ in $H$, the orbit $\left\{T^{n} x: n \geq 0\right\}$ is dense in $H$. We show the existence of a hypercyclic operator-in fact, a bilateral weighted shift-whose adjoint is also hypercyclic. This answers positively a question of D. A. Herrero.
\end{abstract}

\section{INTRODUCTION}

Let $H$ be a complex, separable, infinite-dimensional Hilbert space. Let $B(H)$ denote the bounded linear operators acting on $H$. Let $T \in B(H)$ and $x \in H$. The orbit of $x$ under $T$ is

$$
\operatorname{Orb}(T, x)=\left\{x, T x, T^{2} x, \ldots\right\} .
$$

$T$ is said to be cyclic if there exists $y \in H$ such that the linear span of $\operatorname{Orb}(T, y)$ is dense in $H ; T$ is hypercyclic if $\operatorname{Orb}(T, y)$ itself is dense in $H$. In this last case, $y$ is hypercyclic for $T$ (in [2] such a $y$ is called universal, and in [4] such a $y$ is called orbital).

In a recent paper [7], D. A. Herrero completely characterized the closure of the class of hypercyclic operators in $B(H)$ in terms of spectral properties. His proof is based on previous work of C. Kitai [10], Godefroy and Shapiro [3], and Herrero [5] and [6]. Also a key result that he uses is the similarity orbit theorem [1]. In [7], Herrero raises several questions. The purpose of this note is to answer positively Question 2: "Does there exist $T \in B(H)$ such that both $T$ and $T^{*}$ are hypercyclic?" We also answer in the affirmative Question 3: "Does there exist a hypercyclic backward shift such that $b_{0} b_{1} \cdots b_{k-1}$ does not tend to infinity? (Here the $b_{i}$ 's are the weights of the shift.)"

Gethner and Shapiro proved in [2] that if the weights $b_{k} \geq 1$ and $b_{0} \cdots b_{k}$ go to infinity, then the backward shift is hypercyclic. In [7], it is shown that the condition $b_{k} \geq 1$ can be dropped. Observe that if $U$ is the unilateral (unweighted) shift, $2 U^{*}$ is hypercyclic, while $2 U$ is not.

Received by the editors January 2, 1990 and, in revised form, April 18, 1990.

1980 Mathematics Subject Classification (1985 Revision). Primary 47B99; Secondary 47B37.

Key words and phrases. Cyclic vectors, hypercyclic vectors and operators, weighted shifts. 


\section{THE EXAMPLE}

Let $\left\{e_{k}: k \in Z\right\}$ be an orthonormal basis for the Hilbert space $H$. Let $S \in B(H)$ be defined by $S e_{k}=e_{k+1}$; i.e., $S$ is a bilateral (unweighted) shift.

Assume that $\left\{z_{k}=\sum_{i=-p_{k}}^{p_{k}} z_{k}(i) e_{i}: k \in N\right\}^{-}=H$. For $\left\{n_{i}: i= \pm 1, \pm 2\right.$, $\ldots\}$, to be defined later, and so that $\cdots<n_{-2}<n_{-1}<0<n_{1}<n_{2}<\ldots$, we define a sequence of weights:

$$
b_{k}= \begin{cases}2 & \text { if } k \neq n_{i}, \\ 2^{-2 n_{i+1}} & \text { if } k=n_{-i} \text { and } i>0, \\ 2^{2 n_{-i}} & \text { if } k=n_{i} \text { and } i>0 .\end{cases}
$$

We will construct $T \in B(H)$ such that $T$ and $T^{*}$ are both hypercyclic; the polar decomposition of $T$ will be

$$
T=S^{*} P, \quad \text { where } P e_{k}=b_{k} e_{k} .
$$

Let $-n_{1}=n_{-1}$ be such that $n_{1}>2\left(p_{1}+p_{2}\right)$ and $2^{-n_{1}+p_{1}+1}\left\|z_{1}\right\|<1 / 2$. Let $x_{1}=2^{-n_{1}+p_{1}+1} S^{n_{1}-p_{1}-1}\left(z_{1}\right)$ and $y_{1}=2^{-n_{1}+p_{1}+1}\left(S^{*}\right)^{n_{1}-p_{1}-1}\left(z_{1}\right)$.

Assume that we have already chosen $n_{i}, n_{-i}, x_{i}$, and $y_{i}$ for $1 \leq i \leq j$,

$$
\begin{gathered}
x_{i} \in \operatorname{span}\left\{e_{k}: n_{i}-2 p_{i}-1 \leq k \leq n_{i}-1\right\}, \\
y_{i} \in \operatorname{span}\left\{e_{k}: n_{-i}+1 \leq k \leq n_{-i}+2 p_{i}+1\right\},
\end{gathered}
$$

so that, for $1<k \leq j$,

$$
\begin{gathered}
n_{k}>2\left(n_{k-1}+\left|n_{-k+1}\right|+\sum_{i=1}^{k+1} p_{i}\right), \\
\left|n_{-k}\right|>2\left(\left|n_{-k+1}\right|+n_{k}+\sum_{i=1}^{k+1} p_{i}\right), \\
\left\|x_{k}\right\|<2^{-2 n_{k-1}}, \\
\left\|y_{k}\right\|<2^{2 n_{-k+1}} .
\end{gathered}
$$

We are now ready to choose $n_{j+1}$ and $x_{j+1}$; next we will choose $n_{-j-1}$ and $y_{j+1}$. Recall that $b_{k}=2$ if $n_{j}<k \leq n_{j}+2 p_{j+1}$. Set

$$
u_{j+1}=\sum_{i=-p_{j+1}}^{p_{j+1}}\left(\prod_{r=i+1}^{n_{j}+p_{j+1}+i} b_{r}\right)^{-1} z_{j+1}(i) e_{n_{j}+p_{j+1}+i} .
$$

Let $n_{j+1}$ be a positive integer satisfying

$$
\begin{gathered}
n_{j+1}>2\left(n_{j}+\left|n_{-j}\right|+\sum_{i=1}^{j+2} p_{i}\right), \\
2^{-n_{j+1}+2 p_{j+1}}\left\|u_{j+1}\right\|<2^{-3 n_{j}-1} .
\end{gathered}
$$


Set

$$
x_{j+1}=2^{-n_{j+1}+n_{j}+2 p_{j+1}+1} S^{n_{j+1}-n_{j}-2 p_{j+1}-1}\left(u_{j+1}\right) .
$$
Let

Having defined $n_{j+1}$ and $x_{j+1}$, we now proceed to define $n_{-j-1}$ and $y_{-j-1}$.

$$
v_{j+1}=\sum_{i=-p_{j+1}}^{p_{j+1}}\left(\prod_{r=n_{-j}-p_{j+1}+i}^{i} b_{r}\right)^{-1} z_{j+1}(i) e_{n_{-j}-p_{j+1}+i-1} .
$$

Let $n_{-j-1}$ be a negative integer satisfying

$$
\begin{gathered}
\left|n_{-j-1}\right|>2\left(n_{j+1}+\left|n_{-j}\right|+\sum_{i=1}^{j+2} p_{i}\right) \\
2^{n_{-j-1}+2 p_{j+1}}\left\|v_{j+1}\right\|<2^{3 n_{-j}-2} .
\end{gathered}
$$

Set

$$
y_{j+1}=2^{n_{-j-1}-n_{-j}+2 p_{j+1}+2}\left(S^{*}\right)^{-n_{-j-1}+n_{-j}-2 p_{j+1}-2}\left(v_{j+1}\right) .
$$

Define $x=\sum_{k=1}^{\infty} x_{k}$ and $y=\sum_{k=1}^{\infty} y_{k}$. Since $\left\{n_{i}: i \in Z \backslash\{0\}\right\}$ has been specified, the weights are defined by (1), and $T$ is defined by (2). We will show that $x$ is hypercyclic for $T$ and that $y$ is hypercyclic for $T^{*}$. Observe that definitions (9) and (12) imply that

$$
T^{n_{j}-p_{j}-1}\left(x_{j}\right)=z_{j}
$$

while definitions (13) and (16) imply that

$$
\left(T^{*}\right)^{-n_{-j}-p_{j}-1}\left(y_{j}\right)=z_{j} \text {. }
$$

Let $1 \leq k<j$. Definition (1) and inequality (5) imply that

$$
\left\|T^{n_{j}-p_{j}-1}\left(x_{k}\right)\right\|<2^{n_{j}-p_{j}} b_{n_{-j+1}}\left\|x_{k}\right\|<2^{-n_{j}}\left\|x_{k}\right\| \text {. }
$$

On the other hand, if $k>j$, inequality (11) and the definition of $x_{k}$ (see (12)) imply that

$$
\left\|T^{n_{j}-p_{j}-1}\left(x_{k}\right)\right\|<2^{n_{j}-p_{j}}\left\|x_{k}\right\|<2^{-2 n_{k-1}+n_{j}} .
$$

These last two estimates, together with (17), imply that

$$
\begin{aligned}
\left\|T^{n_{j}-p_{j}-1}(x)-z_{j}\right\| & <\sum_{k=1}^{j-1}\left\|T^{n_{j}-p_{j}-1}\left(x_{k}\right)\right\|+\sum_{k>j}\left\|T^{n_{j}-p_{j}-1}\left(x_{k}\right)\right\| \\
& <2^{-n_{j}}\left(\sum_{k=1}^{j-1}\left\|x_{k}\right\|\right)+\sum_{k>j} 2^{-2 n_{k-1}+n_{j}} .
\end{aligned}
$$

Thus, from inequalities (5) and (7), it follows that the last quantity converges to zero when $j$ goes to infinity. 
A similar argument (but using (6), (15), (16), and (18) instead of (5), (11), (12), and (17)) yields the result that $\left(T^{*}\right)^{-n_{-j}-p_{j}-1}(y)-z_{j}$ converges to zero when $j$ goes to infinity. This concludes the proof that $T$ and $T^{*}$ are hypercyclic operators.

Remark 1. The compression of $T$ to the subspace spanned by $\left\{e_{k}: k \geq 0\right\}$ answers Question 3 of [7] positively.

Remark 2. D. A. Herrero (personal communication) has observed the following:

(a) Since the representing matrix of $T$ with respect to $\left\{e_{i}: i \in Z\right\}$ has real entries, an unpublished result of Deddens (see [8]) says that $T \oplus T^{*}$ is not hypercyclic; this answers Question 4 of [7] negatively.

(b) Up to now, all the hypercyclic operators have satisfied the Kitai-GethnerShapiro (K-G-S) condition: If $L \in B(H)$ has a right inverse $M$ and a dense subset $D$ of $H$ so that $\left\|L^{n}(x)\right\|$ and $\left\|M^{n}(x)\right\|$ go to zero for every $x \in D$, then $L$ has a hypercyclic vector (here $H$ is separable, see [2, Theorem 2.2]). It is clear that if two operators satisfy the K-G-S condition, then the direct sum also satisfies it, and therefore the direct sum has to be hypercyclic. Thus either $T$ or $T^{*}$ does not satisfy the K-G-S condition.

Remark 3. The question "Is there any hypercyclic operator $L$ such that $\operatorname{Per} L=$ $\{x \in H: \operatorname{Orb}(L, x)$ is periodic $\}$ is a nontrivial finite dimensional space?" [7, Question 7] can also be answered positively. This question relates to [7, Proposition 4.7]. There it is shown that, if $L$ is hypercyclic, either $(\operatorname{Per} L)^{-}=H$ or $(\operatorname{Per} L)^{-}$has infinite codimension in $H$; it is also shown that $(\operatorname{Per} L)^{-}=H$ for all $L$ in a dense subset of the class of hypercyclic operators.

There exists $A=\left(\begin{array}{ll}1 & F \\ 0 & G\end{array}\right) \in B(C \oplus H), A$ hypercyclic, such that $\operatorname{Per} A=$ $C \oplus\{0\} . G$ is a unilateral weighted shift and $F$ is a linear functional. (It is even possible to pick $G$ so that $G$ and $G^{*}$ are both hypercyclic; however, $A^{*}$ cannot be hypercyclic since $A$ has an eigenvector (see [7]).) Herrero and Wang [9] have also answered Question 7 positively as a corollary of their proof of [7, Conjecture 1]. Originally, we did not plan to give details of the construction of $A$, but the referee suggested that it would be more useful to do so.

Let $\left\{e_{n}: n \geq 0\right\}$ be an orthogonal basis for $H$. Given $\left\{\beta_{j} \oplus z_{j}: z_{j}=\right.$ $\left.\sum_{i=0}^{p_{j}} z_{j}(i) e_{i}, j \in N\right\}^{-}=C \oplus H$, it is possible to define inductively four sequences $r_{j}, n_{j}, \alpha_{j}$, and $g_{j}$ so that the functional $F$ is represented by the vector

$$
u=\sum_{j=1}^{\infty} \alpha_{j} e_{n_{j-1}+p_{j}+3},
$$

while $G\left(e_{0}\right)=0$ and $G\left(e_{i+1}\right)=g_{i} e_{i}$. The sequences $r_{j}, n_{j}$ satisfy the relations

$$
\begin{aligned}
n_{j+1} & =2\left(r_{j+1}+p_{j+1}\right)+n_{j}+3, \\
r_{j} & >3\left(\sum_{i \leq j-1} n_{i}+\sum_{i=1}^{j+1} p_{i}\right) .
\end{aligned}
$$


The weight sequence $g_{i}$ satisfies:

$$
g_{i}= \begin{cases}2^{-2 n_{k}} & \text { if } i=n_{k} \quad\left(n_{0}=0\right), \\ 1 & \text { if } n_{k}+1 \leq i \leq 1+n_{k}+p_{k+1}, \\ 1 & \text { if } 1 \leq k \text { and } n_{k}-p_{k}<i \leq n_{k}-1, \\ 2^{-r_{k+1}} & \text { if } i=n_{k}+p_{k+1}+2 \\ 2 & \text { otherwise. }\end{cases}
$$

One can then choose a hypercyclic vector for $A$ of the form

$$
0 \oplus x=0 \oplus \sum_{j=1}^{\infty} x_{j},
$$

where $x_{j} \in \operatorname{span}\left\{e_{i}: n_{j}-p_{j} \leq i \leq n_{j}\right\}$. The $x_{j}$ 's are constructed by using auxiliary vectors $v_{j}$. We show how $x_{1}$ and $x_{j+1}$ are obtained from $v_{1}$ and $v_{j+1}$, respectively. This will put in evidence how $r_{j}, n_{j}$, and $\alpha_{j}$ are interrelated.

If $v=\sum_{i=0}^{m} v(i) e_{i}$, we denote $\sum_{i=0}^{m} v(i)$ by $h(v)$. The vector $v_{1}=$ $\sum_{i=0}^{p_{1}} v_{1}(i) e_{i}$ satisfies

$$
\left\|v_{1}-z_{1}\right\| \leq 1 / 2
$$

and

$$
h\left(v_{1}\right) \neq 0
$$

$r_{1}$ is chosen such that

$$
2^{-r_{1}}\left\|v_{1}\right\| \leq 1 \text { and }\left|\beta_{1} /\left(h\left(v_{1}\right) 2^{r_{1}}\right)\right| \leq 1 / 2
$$

Then $\alpha_{1}$ and $x_{1}$ satisfy

$$
\alpha_{1}=\beta_{1} /\left(h\left(v_{1}\right) 2^{r_{1}}\right) \text { and } x_{1}=2^{-r_{1}} U^{n_{1}-p_{1}}\left(v_{1}\right),
$$

where $U$ is the unilateral shift with respect to $\left\{e_{i}: i \geq 0\right\}$. The vector $v_{k+1} \in$ $\operatorname{span}\left\{e_{i}: n_{k}+2 \leq i \leq n_{k}+p_{k+1}+2\right\}$ and is such that

$$
\left\|G^{n_{k}+2}\left(v_{k+1}\right)-z_{k+1}\right\| \leq 2^{-k-1} \text { and } h\left(v_{k+1}\right) \neq 0 .
$$

The numbers $r_{k+1}$ satisfy

$$
\left\|v_{k+1}\right\| \leq 2^{-2 n_{k}+r_{k+1}},
$$

and if

$$
\alpha_{k+1}=\left(\beta_{k+1}-\beta_{k}-\left[A^{n_{k}+2}\left(0 \oplus v_{k+1}\right), 1 \oplus 0\right]\right) /\left(h\left(v_{k+1}\right) 2^{r_{k+1}}\right),
$$

then $\left|\alpha_{k+1}\right| \leq 2^{-k-1}$. (Here $\beta_{k} \oplus 0=A^{n_{k}+2}\left(0 \oplus \sum_{j=1}^{k} x_{j}\right)$.) Finally,

$$
x_{k+1}=2^{-r_{k+1}} U^{n_{k+1}-n_{k}-p_{k+1}-2}\left(v_{k+1}\right) \text {. }
$$

(Observe that $x_{k+1}$ has been constructed so that

$$
\left.A^{n_{k+1}-n_{k}-p_{k+1}-2}\left(0 \oplus x_{k+1}\right)=2^{r_{k+1}} h\left(v_{k+1}\right) \alpha_{k+1} \oplus v_{k+1} .\right)
$$


To show that $0 \oplus x$ is hypercyclic, it is enough to verify that

$$
\lim _{k \rightarrow \infty}\left\|\left(\beta_{k} \oplus z_{k}\right)-A^{n_{k}-p_{k}}(0 \oplus x)\right\|=0 .
$$

On the other hand, since $g_{0} g_{1} \cdots g_{n_{k}} \leq 2^{-n_{k}}$, the unit circle does not intersect the point spectrum of $G$. Thus $\operatorname{dim}(\operatorname{Per}(A))=1$.

\section{ACKNOWLEDGMENT}

I would like to thank Professor Domingo Herrero for sending me his article [7], which is the origin of the present paper.

Added in proof. In a paper in preparation by D. A. Herrero and the author, conditions for hypercyclicity of operator-valued weighted shifts are studied. In particular, hypercyclic weighted shifts are characterized in terms of their weights. As a consequence of this characterization, the hypercyclic bilateral weighted shifts whose adjoints are also hypercyclic are identified.

\section{REFERENCES}

1. C. Apostol, L. A. Fialkow, D. A. Herrero, and D. Voiculescu, Approximation of Hilbert space operators, Vol. II, Research Notes in Math. 102, Pitman, Boston, 1984.

2. R. M. Gethner and J. H. Shapiro, Universal vectors for operators on spaces of holomorphic functions, Proc. Amer. Math. Soc. 100 (1987), 281-288.

3. G. Godefroy and J. H. Shapiro, Operators with dense, invariant cyclic vector manifolds, J. Funct. Anal. (to appear).

4. I. Halperin, C. Kitai, and P. Rosenthal, On orbits of linear operators, J. London Math. Soc. 31 (1985), 561-565.

5. D. A. Herrero, The diagonal entries in the formula ' Quasitriangular - compact $=$ triangular' and restrictions of quasitriangularity, Trans. Amer. Math. Soc. 289 (1987), 1-42.

6. __ Spectral pictures of operators in the Cowen-Douglas class $B_{n}(\Omega)$ and its closure, $\mathrm{J}$. Operator Theory 18 (1988), 213-222.

7. _ Limits of hypercyclic and supercyclic operators, preprint.

8. D. A. Herrero and W. R. Wogen, On the multiplicity of $T \oplus T \oplus \cdots \oplus T$, Rocky Mountain J. Math. (to appear).

9. D. A. Herrero and Z. Y. Wang, Compact perturbations of hypercyclic and supercyclic operators, Indiana Univ. Math. J. (to appear).

10. C. Kitai, Invariant closed sets for linear operators, Thesis, Univ. of Toronto, 1982.

Department of Mathematics, State University of New York at New Paltz, New Paltz, NEW YORK 12561

Current address: Departamento de Matemáticas, Universidad de Puerto Rico, Mayaguez, Puerto Rico 00708 